\title{
Gauge-invariant inflaton in the minimal supersymmetric standard model
}

\author{
Rouzbeh Allahverdi, ${ }^{1,2}$ Kari Enqvist, ${ }^{3}$ Juan Garcia-Bellido, ${ }^{4}$ and Anupam Mazumdar ${ }^{5}$ \\ ${ }^{1}$ Perimeter Institute for Theoretical Physics, Waterloo, ON, N2L 2Y5, Canada \\ ${ }^{2}$ Department of Physics and Astronomy, McMaster University, Hamilton, ON, L8S 4M1, Canada \\ ${ }^{3}$ Department of Physical Sciences, University of Helsinki, and Helsinki Institute of Physics, \\ P.O. Box 64, FIN-00014, Helsinki, Finland \\ ${ }^{4}$ Departamento de Física Teórica C-XI, Universidad Autónoma de Madrid, Cantoblanco, 28049 Madrid, Spain \\ ${ }^{5}$ NORDITA, Blegdamsvej-17, Copenhagen-2100, Denmark
}

(Received 3 June 2006; published 9 November 2006)

\begin{abstract}
We argue that all the necessary ingredients for successful inflation are present in the flat directions of the Minimally Supersymmetric Standard Model. We show that out of many gauge-invariant combinations of squarks, sleptons, and Higgs bosons, there are two directions, LLe and udd, which are promising candidates for the inflaton. The model predicts more than $10^{3} e$-foldings, with an inflationary scale of $H_{\text {inf }} \sim \mathcal{O}(1-10) \mathrm{GeV}$, provides a tilted spectrum with an amplitude of $\delta_{H} \sim 10^{-5}$ and a negligible tensor perturbation. The temperature of the thermalized plasma could be as low as $T_{r h} \sim \mathcal{O}(1-10) \mathrm{TeV}$. Parts of the inflaton potential can be determined independently of cosmology by future particle physics experiments.
\end{abstract}

DOI: 10.1103/PhysRevLett.97.191304

PACS numbers: $98.80 . \mathrm{Cq}, 12.60 . \mathrm{Jv}$

The one crucial ingredient still missing in the otherwise highly successful theory of primordial inflation is its natural embedding within particle physics, particularly to the Standard Model (SM) or its extensions. In almost all models of inflation, the inflaton is treated as a SM gauge singlet and sometimes a complete gauge singlet whose origin and couplings are chosen ad-hoc just to fit the observed cosmological data [1] without bothering about the relation to the observed particle contents of the universe [2]. The only exception is the large $N$ inflationary models [3], where the gauge-invariant quasiflat directions of $\mathrm{SO}(\mathrm{N})$ are responsible for driving assisted inflation at sub-Planckian vacuum expectation values (VEVs)[4].

The Minimal Supersymmetric Standard Model (MSSM) is a well motivated extension of the SM with many cosmological consequences [5]. MSSM has nearly 300 gaugeinvariant flat directions made up of squarks, sleptons, and Higgs bosons [6,7], whose potentials are vanishing in the supersymmetric limit. However, they are lifted by a soft supersymmetry (SUSY) breaking mass term, the trilinear $A$-term, and by nonrenormalizable superpotential corrections at scales below the fundamental scale, which we take to be the Planck scale, $M_{P}=2.4 \times 10^{18} \mathrm{GeV}$.

Although in the MSSM one usually also relies on a gauge singlet inflaton, in the present Letter we shall show that there are two flat directions which may serve as a low-scale inflaton; we thus provide the first example of MSSM inflation occurring at scales well below the Planck scale and involving a sub-Planckian VEV of the flat direction. Thus, we argue that all the inflationary ingredients are present within MSSM and do not necessarily require anything beyond MSSM. In particular, we show that the MSSM inflaton is capable of creating the right amplitude of the scalar perturbations with a tilted spectrum.
Moreover, in the present model, certain properties of the inflaton are in principle testable in future collider experiment, such as Large Hadron Collider (LHC) [8].

Let us begin by considering a flat direction $\phi$ with a nonrenormalizable superpotential term,

$$
W=\frac{\lambda_{n}}{n} \frac{\Phi^{n}}{M_{P}^{n-3}},
$$

where $\Phi$ is the superfield which contains the flat direction. Within MSSM, all the flat directions are lifted by $n=9$ nonrenormalizable operators [7]. Together with the corresponding $A$-term and the soft mass term, it gives rise to the following scalar potential for $\phi$ :

$$
V=\frac{1}{2} m_{\phi}^{2} \phi^{2}+A \cos \left(n \theta+\theta_{A}\right) \frac{\lambda_{n} \phi^{n}}{n M_{P}^{n-3}}+\lambda_{n}^{2} \frac{\phi^{2(n-1)}}{M_{P}^{2(n-3)}},
$$

where $m_{\phi}$ is the soft SUSY breaking mass for $\phi$. Here $\phi$ and $\theta$ denote the radial and the angular coordinates of the complex scalar field $\Phi=\phi \exp [i \theta]$, respectively, while $\theta_{A}$ is the phase of the $A$-term (thus $A$ is a positive quantity with a dimension of mass). Note that the first and third terms in Eq. (2) are positive definite, while the $A$-term leads to a negative contribution along the directions where $\cos (n \theta+$ $\left.\theta_{A}\right)<0$.

The maximum impact from the $A$-term is obtained when $\cos \left(n \theta+\theta_{A}\right)=-1$ (which occurs for $n$ values of $\theta$ ). Along these directions, $V$ has a secondary minimum at

$$
\phi=\phi_{0} \sim\left(m_{\phi} M_{P}^{n-3}\right)^{1 / n-2} \ll M_{P}
$$

(the global minimum is at $\phi=0$ ), provided that

$$
A^{2} \geq 8(n-1) m_{\phi}^{2} \text {. }
$$


At this minimum, the curvature of the potential along the radial direction is $+m_{\phi}^{2}$ (it is easy to see that the curvature is positive along the angular direction, too), and the potential reduces to $V \sim m_{\phi}^{2} \phi_{0}^{2} \sim m_{\phi}^{2}\left(m_{\phi} M_{P}^{n-3}\right)^{2 /(n-2)}$. If $A$ is too large, the secondary minimum will be deeper than the one at the origin, and hence becomes the true minimum. However, this is phenomenologically unacceptable, since such a minimum would break charge and/or color [6]. Now consider the situation where the flat direction is trapped in the shallow minimum $\phi_{0}$. If its potential energy, $V$, dominates the total energy density of the Universe, a period of inflation is obtained. The Hubble expansion rate during inflation will then be

$$
H_{\mathrm{inf}} \sim \frac{m_{\phi} \phi_{0}}{M_{P}} \sim m_{\phi}\left(\frac{m_{\phi}}{M_{P}}\right)^{1 /(n-2)} .
$$

Note that $H_{\text {inf }} \ll m_{\phi}$. This implies that the potential is too steep at the false minimum and $\phi$ cannot climb over the barrier which separates the two minima just by the help of quantum fluctuations during inflation. The situation is essentially the same as in the old inflation scenario [9] with no graceful exit from inflation.

An interesting but rather trivial observation is that the potential barrier disappears when the inequality in Eq. (4) is saturated, i.e., when

$$
A^{2}=8(n-1) m_{\phi}^{2} .
$$

Then both the first and second derivatives of $V$ vanish at $\phi_{0}$, i.e., $V^{\prime}\left(\phi_{0}\right)=0, V^{\prime \prime}\left(\phi_{0}\right)=0$, and the potential becomes very flat along the real direction. Around $\phi_{0}$, the field is stuck in a plateau with potential energy

$$
\begin{aligned}
& V\left(\phi_{0}\right)=\frac{(n-2)^{2}}{2 n(n-1)} m_{\phi}^{2} \phi_{0}^{2}, \\
& \phi_{0}=\left(\frac{m_{\phi} M_{P}^{n-3}}{\lambda_{n} \sqrt{2 n-2}}\right)^{1 /(n-2)} .
\end{aligned}
$$

However, although the second derivative of the potential vanishes, the third does not; instead,

$$
V^{\prime \prime \prime}\left(\phi_{0}\right)=2(n-2)^{2} \frac{m_{\phi}^{2}}{\phi_{0}} .
$$

Around $\phi=\phi_{0}$, we can thus expand the potential as $V(\phi)=V\left(\phi_{0}\right)+(1 / 3 !) V^{\prime \prime \prime}\left(\phi_{0}\right)\left(\phi-\phi_{0}\right)^{3}$. Hence, in the range $\quad\left[\phi_{0}-\Delta \phi, \phi_{0}+\Delta \phi\right]$, where $\Delta \phi \sim$ $H_{\text {inf }}^{2} / V^{\prime \prime \prime}\left(\phi_{0}\right) \sim\left(\phi_{0}^{3} / M_{P}^{2}\right) \gg H_{\text {inf }}$, the real direction has a flat potential.

We can now solve the equation of motion for the $\phi$ field in the slow-roll approximation, $3 H \dot{\phi}=-(1 / 2) V^{\prime \prime \prime}\left(\phi_{0}\right) \times$ $\left(\phi-\phi_{0}\right)^{2}$. Note that the field only feels the third derivative of the potential. Thus, if the initial conditions are such that the flat direction starts in the vicinity of $\phi_{0}$ with $\dot{\phi} \approx$ 0 , then a sufficiently large number of $e$-foldings of the scale factor can be generated. In fact, quantum fluctuations along the tachyonic direction [10] will drive the field towards the minimum. However, quantum diffusion is stronger than the classical force, $H_{\mathrm{inf}} / 2 \pi>\dot{\phi} / H_{\mathrm{inf}}$, for

$$
\frac{\left(\phi_{0}-\phi\right)}{\phi_{0}} \lesssim\left(\frac{m_{\phi} \phi_{0}^{2}}{M_{P}^{3}}\right)^{1 / 2},
$$

but from then on, the evolution is determined by the usual slow roll.

A rough estimate of the number of $e$-foldings is then given by

$$
\mathcal{N}_{e}(\phi)=\int \frac{H d \phi}{\dot{\phi}} \simeq \frac{\phi_{0}^{3}}{2 n(n-1) M_{P}^{2}\left(\phi_{0}-\phi\right)},
$$

where we have assumed $V^{\prime}(\phi) \sim\left(\phi-\phi_{0}\right)^{2} V^{\prime \prime \prime}\left(\phi_{0}\right)$ (this is justified since $\left.V^{\prime}\left(\phi_{0}\right) \sim 0, V^{\prime \prime}\left(\phi_{0}\right) \sim 0\right)$. Note that the initial displacement from $\phi_{0}$ cannot be smaller than $H_{\text {inf }}$, due to the uncertainty from quantum fluctuations.

Inflation ends when $\epsilon \sim 1$, or

$$
\frac{\left(\phi_{0}-\phi\right)}{\phi_{0}} \sim\left(\frac{\phi_{0}}{2 n(n-1) M_{P}}\right)^{1 / 2} .
$$

After inflation, the coherent oscillations of the flat direction excite the MS(SM) degrees of freedom and reheat the universe.

Let us now identify the possible MSSM inflaton candidates. Recall first that the highest order operators which give a nonzero $A$-term are those with $n=6$. This happens for flat directions represented by the gauge-invariant monomials

$$
\phi=\mathbf{L}_{i} \mathbf{L}_{j} \mathbf{e}_{k} ; \quad \phi=\mathbf{u d}_{i} \mathbf{d}_{j}
$$

The flatness of the potential require that $i \neq j \neq k$ in the former and $i \neq j$ in the latter. For $n=6$ and $m_{\phi} \sim 1 \mathrm{TeV}$, as in the case of weak scale supersymmetry breaking, we find the following generic results:

(a) Sub-Planckian VEVs. - In an effective field theory where the Planck scale is the cutoff, the inflationary potential can be trusted only below the Planck scale, usually a challenge for model building [11]. In our case, the flat direction VEV is sub-Planckian for the nonrenormalizable operator $n=6$, i.e., $\phi_{0} \sim 1-3 \times 10^{14} \mathrm{GeV}$ for $m_{\phi} \sim$ $1-10 \mathrm{TeV}$, while the vacuum energy density ranges $V \sim$ $10^{34}-10^{38}(\mathrm{GeV})^{4}$ (in this Letter we assume $\lambda_{n}=1$; generically $\lambda_{n} \leq 1$, but its precise value depends on the nature of high energy physics).

(b) Low-scale inflation. - Although it is extremely hard to build an inflationary model at low scales, for the energy density stored in the MSSM flat direction vacuum, the Hubble expansion rate comes out as low as $H_{\text {inf }} \sim$ $1-10 \mathrm{GeV}$. It might be possible to lower the scale of inflation further to the electroweak scale.

(c) Enough e-foldings. -At low scales, $H_{\text {inf }} \sim$ $\mathcal{O}(1) \mathrm{GeV}$, the number of $e$-foldings, $\mathcal{N}_{\mathrm{COBE}}$, required 
for the observationally relevant perturbations, is much less than 60 [12]. In fact, the number depends on when the Universe becomes radiation dominated (note that full thermalization is not necessary as it is the relativistic equation of state which matters).

If the inflaton decays immediately after the end of inflation, which has a scale $V \sim 10^{36}(\mathrm{GeV})^{4}$, we obtain $\mathcal{N}_{\mathrm{COBE}} \sim 47$ [12]. The relevant number of $e$-foldings could be greater if the scale of inflation becomes larger. For instance, if $m_{\phi} \sim 10 \mathrm{TeV}$, and $V \sim 10^{38}(\mathrm{GeV})^{4}$, we have $\mathcal{N}_{\mathrm{COBE}} \sim 50$. For the MSSM flat direction lifted by $n=6$ nonrenormalizable operators, we obtain the total number of $e$-foldings as

$$
\mathcal{N}_{e} \sim\left(\frac{\phi_{0}^{2}}{m_{\phi} M_{P}}\right)^{1 / 2} \sim 10^{3},
$$

computed from the end of diffusion, see Eq. (10). This bout of inflation is sufficiently long to produce a patch of the Universe with no dangerous relics. Domains initially closer to $\phi_{0}$ will enter self-reproduction in eternal inflation.

Let us now consider adiabatic density perturbations. Despite the low inflationary scale $H_{\text {inf }} \sim \mathcal{O}(1) \mathrm{GeV}$, the flat direction can generate adequate density perturbations as required to explain the COBE normalization. This is due to the extreme flatness of the potential (recall that $V^{\prime}=0$ ), which causes the velocity of the rolling flat direction to be extremely small. Thus we find an amplitude of

$$
\delta_{H} \simeq \frac{1}{5 \pi} \frac{H_{\mathrm{inf}}^{2}}{\dot{\phi}} \sim \frac{m_{\phi} M_{P}}{\phi_{0}^{2}} \mathcal{N}_{\mathrm{COBE}}^{2} \sim 10^{-5},
$$

for $m_{\phi} \sim 10^{3}-10^{4} \mathrm{GeV}$, where $\phi_{0}$ is given by Eq. (7). In the above expression we have used the slow-roll approximation $\dot{\phi} \simeq-V^{\prime \prime \prime}\left(\phi_{0}\right)\left(\phi_{0}-\phi\right)^{2} / 3 H_{\text {inf }}$, and Eq. (11). Note the importance of the $n=6$ operators lifting the flat directions LLe and udd. Higher order operators would have allowed for larger VEVs and a large $\phi_{0}$, therefore leaving the amplitude of the perturbations too low.

The spectral tilt of the power spectrum is not negligible because, although $\epsilon \sim 1 / \mathcal{N}_{\text {COBE }}^{4} \ll 1$, the parameter $\eta=$ $-2 / \mathcal{N}_{\mathrm{COBE}}$ and thus

$$
\begin{gathered}
n_{s}=1+2 \eta-6 \epsilon \simeq 1-\frac{4}{\mathcal{N}_{\mathrm{COBE}}} \sim 0.92, \\
\frac{d n_{s}}{d \ln k}=-\frac{4}{\mathcal{N}_{\mathrm{COBE}}^{2}} \sim-0.002,
\end{gathered}
$$

which agrees with the current WMAP 3-years' data within $2 \sigma$ [1], while there are essentially no tensor modes. Note that the tilt can be enhanced to match the central value of the Wilkinson Microwave Anisotropy Probe (WMAP) 3years' data while tuning $\lambda_{n}$ to values lower than 1 .

Recall that quantum loops result in a logarithmic running of the soft supersymmetry breaking parameters $m_{\phi}$ and $A$. One might then worry about their impact on Eq. (6) and the success of inflation. Note however that the only implication is that one must use the VEV-dependent values of $m_{\phi}(\phi)$ and $A(\phi)$ in Eq. (6) and in determining $\phi_{0}$. We have checked that the crucial ingredient for a successful inflation, i.e., having a very flat potential such that $V^{\prime}\left(\phi_{0}\right)=V^{\prime \prime}\left(\phi_{0}\right)=0$, will remain unchanged under quantum corrections.

After the end of inflation, the flat direction eventually starts rolling towards its global minimum. The flat direction decays into light relativistic MS(SM) particles which obtain kinetic equilibrium rather quickly [13,14], with the largest temperature of the plasma given by

$$
T_{\max } \sim[V(\phi)]^{1 / 4} \sim\left(H_{\mathrm{inf}} M_{P}\right)^{1 / 2} \sim 10^{8} \mathrm{GeV} .
$$

Although the plasma heats up to a large value due to large momenta of the inflaton decay products, the process of thermalization, which requires chemical equilibrium, can be a slow process [14]. Just to illustrate, we note that within MSSM there are other flat directions orthogonal to Eq. (13) (for an algorithm finding such multidirections, see [15]). These can develop large VEVs and induce large masses to the MSSM quanta, i.e., squarks and sleptons and gauge bosons and gauginos. As a consequence, there will be a kinematical blocking for the inflaton to decay [16] which can delay thermalization as discussed in Refs. $[13,14]$.

The details of thermalization would require involved calculations which are beyond the scope of the present Letter. However, perhaps the best guess is to assume that the flat direction mass gives the lower limit on a temperature, where all the MSSM degrees of freedom are in thermal equilibrium [14]:

$$
T_{r h} \sim m_{\phi} \sim \mathcal{O}(1-10) \mathrm{TeV} .
$$

Note that this temperature is sufficiently high for electroweak baryogenesis [17] and for both thermal and nonthermal cold dark matter production [18]. Affleck-Dine baryogenesis [19] via other MSSM flat directions is also an option. For an example, the fragmentation of udd can generate $Q$-balls [20], which could be responsible for baryogenesis; and the remnant $Q$-balls could act as cold dark matter [5].

Let us also briefly mention an alternative possibility for creating the density perturbations through a curvaton [21]. MSSM flat directions can provide us with curvaton candidates [22] when a flat direction orthogonal to the inflaton, $\varphi$, achieves a large VEV during inflation (provided their potential follows Eq. (2) with $\phi \rightarrow \varphi$ while satisfying Eq. (6)). If the VEV is such that the amplitude of the perturbations is $\delta=H_{\text {inf }} / \varphi_{*} \sim 10^{-5}$, and the fluctuations are Gaussian, then such an orthogonal direction can act as a curvaton. A promising curvaton candidate is $\mathbf{H}_{u} \mathbf{Q}_{2} \mathbf{u}_{2}$ (indices refer to the generation), if $\mathbf{L L e}$ is the inflaton.

Let us finally turn to the issue of initial conditions. The Universe could begin very hot, with all the relevant degrees 
of freedom excited. As it cools, in a small patch it can achieve an initial state where the flat direction potential is given by Eq. (6) and inflation can begin. This is the simplest possibility, but there are also others, e.g., a running scale/coupling could modify the VEV of $A$ so that the universe is initially stuck in the inflationary minimum at $\phi_{0}$, which is later lifted to become a turning point; or there could be multiple phases of inflation, as has been often argued, see, e.g., Ref. [23], with a last phase driven by the MSSM inflaton.

To summarize, for the first time, a gauge-invariant inflaton candidate has been proposed within MSSM without any inclusion of gauge singlets. The inflaton candidates are the flat directions udd and L Le. Both are lifted by nonrenormalizable operators at $n=6$ level. Lower dimension operators do not provide the $A$-term required for inflation, while higher order operators fail to generate the observed cosmic microwave background fluctuations. The model predicts low-scale inflation, nondetectable gravity waves, and a slight departure from scale invariance. As we have discussed, the conservative estimate for the spectral index is $n_{s} \sim 0.92$, but a small enhancement giving a match to the WMAP 3-year data is possible with a slight tuning of the flat direction coupling strength. Further note that supergravity effects are negligible for $H_{\text {inf }} \ll m_{\phi}$ and therefore do not spoil the predictions. More importantly, there will be no moduli problem in our model. Since $H_{\text {inf }} \ll m_{\phi}$, all moduli will settle at their true minimum during inflation even if their initial displacement is $\mathcal{O}\left(M_{P}\right)$.

The salient feature of the present model is that in principle the properties of the inflaton are now related to the dynamics of baryogenesis and the properties of dark matter. Moreover, some properties of the inflaton potential, such as the soft mass term and the $A$-term, could be determined independently of cosmology by particle physics experiments, possibly already at LHC.

We wish to thank Andrew Liddle and Jaume Garriga for helpful comments. The work of R. A. is supported by the National Sciences and Engineering Research Council of Canada (NSERC). J. G. B. is supported in part by a CICYT Project No. FPA2003-04597. K. E. is supported in part by the Academy of Finland Grant No. 205800.

[1] D. N. Spergel et al., astro-ph/0603449.

[2] For a review, see, e.g., D. H. Lyth and A. Riotto, Phys. Rep. 314, 1 (1999).

[3] R. Brandenberger, P. M. Ho, and H. C. Kao, J. Cosmol. Astropart. Phys. 11 (2004) 011; A. Jokinen and A. Mazumdar, Phys. Lett. B 597, 222 (2004).
[4] A. R. Liddle, A. Mazumdar, and F. E. Schunck, Phys. Rev. D 58, 061301(R) (1998); E. J. Copeland, A. Mazumdar, and N.J. Nunes, Phys. Rev. D 60, 083506 (1999); A. Mazumdar, S. Panda, and A. PerezLorenzana, Nucl. Phys. B614, 101 (2001).

[5] For reviews, see K. Enqvist and A. Mazumdar, Phys. Rep. 380, 99 (2004); M. Dine and A. Kusenko, Rev. Mod. Phys. 76, 1 (2003).

[6] M. Dine, L. Randall, and S. Thomas, Phys. Rev. Lett. 75, 398 (1995); M. Dine, L. Randall, and S. Thomas, Nucl. Phys. B458, 291 (1996).

[7] T. Gherghetta, C. Kolda, and S. P. Martin, Nucl. Phys. B468, 37 (1996).

[8] See for instance: http://hc.web.cern.ch/lhc/.

[9] A. H. Guth, Phys. Rev. D 23, 347 (1981).

[10] G. N. Felder, J. Garcia-Bellido, P. B. Greene, L. Kofman, A. D. Linde, and I. Tkachev, Phys. Rev. Lett. 87, 011601 (2001); G. N. Felder, L. Kofman, and A. D. Linde, Phys. Rev. D 64, 123517 (2001).

[11] G. Lazarides and Q. Shafi, Phys. Lett. B 308, 17 (1993); S. Kasuya, T. Moroi, and F. Takahashi, Phys. Lett. B 593, 33 (2004).

[12] A. R. Liddle and S. M. Leach, Phys. Rev. D 68, 103503 (2003).

[13] R. Allahverdi and A. Mazumdar, hep-ph/0505050.

[14] R. Allahverdi and A. Mazumdar, hep-ph/0512227.

[15] K. Enqvist, A. Jokinen, and A. Mazumdar, J. Cosmol. Astropart. Phys. 01 (2004) 008.

[16] R. Allahverdi and A. Mazumdar, hep-ph/0603244.

[17] V. A. Rubakov and M.E. Shaposhnikov, Usp. Fiz. Nauk 166, 493 (1996) [Phys. Usp. 39, 461 (1996)]; J. GarciaBellido, D. Y. Grigoriev, A. Kusenko, and M.E. Shaposhnikov, Phys. Rev. D 60, 123504 (1999).

[18] G. Bertone, D. Hooper, and J. Silk, Phys. Rep. 405, 279 (2005).

[19] I. Affleck and M. Dine, Nucl. Phys. B249, 361 (1985).

[20] A. Kusenko and M.E. Shaposhnikov, Phys. Lett. B 418, 46 (1998); G. R. Dvali, A. Kusenko, and M.E. Shaposhnikov, Phys. Lett. B 417, 99 (1998); K. Enqvist and J. McDonald, Phys. Lett. B 425, 309 (1998); K. Enqvist and J. McDonald, Nucl. Phys. B538, 321 (1999).

[21] K. Enqvist and M. S. Sloth, Nucl. Phys. B626, 395 (2002); D. H. Lyth and D. Wands, Phys. Lett. B 524, 5 (2002); T. Moroi and T. Takahashi, Phys. Lett. B 522, 215 (2001); 539, 303(E) (2002); A. D. Linde and V.F. Mukhanov, Phys. Rev. D 56, R535 (1997).

[22] K. Enqvist, S. Kasuya, and A. Mazumdar, Phys. Rev. Lett. 90, 091302 (2003); K. Enqvist, A. Jokinen, S. Kasuya, and A. Mazumdar, Phys. Rev. D 68, 103507 (2003); R. Allahverdi, K. Enqvist, A. Jokinen, and A. Mazumdar, hep-ph/0603255.

[23] C. P. Burgess, R. Easther, A. Mazumdar, D. F. Mota, and T. Multamaki, J. High Energy Phys. 05 (2005) 067. 